Original Article

\title{
Pilot study to evaluate the efficacy of biotherapy in the treatment of chronic periodontitis: double-blind, placebo-controlled assay
}

\section{Leila Cristina Mourão ${ }^{1,2^{*}}$, Fortune Homsai ${ }^{1}$, Paulo Murillo Neufeld ${ }^{3}$, Roseli Vígio Ribeiro ${ }^{3}$, Antonio Canabarro $^{2,4}$, Carla Holandino ${ }^{1}$}

1 Laboratório Multidisciplinar de Ciências Farmacêuticas, Faculdade de Farmácia, Universidade Federal do Rio de Janeiro - RJ, Brasil. 2 Universidade Veiga de Almeida, Rio de Janeiro - RJ, Brasil., and ABRAH / UFF - Associação Brasileira de Reciclagem Assistência em Homeopatia - Universidade Federal Fluminense. E-mail:* leilamourao@hotmail.com. 3 Faculdade de Farmácia, Universidade Federal do Rio de Janeiro - RJ, Brasil. 4 Faculdade de Odontologia, Universidade Estadual do Rio de Janeiro RJ, Brasil

\begin{abstract}
Background: Periodontitis constitutes a group of infectious diseases, associated with local and systemic etiological factors. Among these factors, the bacterial plaque has been considered the main causal factor. Biotherapies are homeopathic medicines prepared from biological products, such as bacteria and virsuses, and can be used to treat and to prevent infectious diseases [1].
\end{abstract}

Aims: To evaluate the clinical efficacy of the biotherapy as adjuvant in the treatment of chronic periodontal disease.

Methodology: After the Ethics Committee approval, nine patients of both genders and with age ranging from 35 to 70 years old, diagnosed with chronic periodontitis were selected by dentistry professionals from the PeriodontatClinic of the Veiga de Almeida University (Rio de Janeiro, Brazil). They were divided in three groups, with 3 patients each-Group 1- treated with biotherapy poll (BP); Group 2- treated with auto-isotherapy: Group 3 - treated with placebo (ethanol 30\% v/v). Each test solution was used twice a day, in the popolog 6 drops/dose, for 90 days. Before the treatment, the dentists collected two sapples (Da seling the gingival fluid, with a sterile paper, with higher levels of Bleeding on Probing (BOP>5 mm) and Clinical Attachment Level (CAL>3 mm). These materials were transferred to eppendorf tubes containing microbiological medium for 15 days, and after the microbial growth, they were used as start point to the biotherapy compounding. All the solutions were prepared in $30 \mathrm{dH}$, using ethanol $30 \%(\mathrm{v} / \mathrm{v}$ ) as vehicle, at Homeopathic Pharmacy (Faculdade de Farmácia, UFRJ), following the Brazilian Homeopathic Pharmacopea [2]. After the samples preparation, all patients were submitted a clinical evaluation and received the specific solutions. By the end of treatment scheme, the patients returned and were submitted a new clinical evaluation, as well as to the sample collection, following the same parameters used in the initial phase $(\mathrm{BOP}>5 \mathrm{~mm}$; $\mathrm{CAL}>3 \mathrm{~mm})$.

Results and Discussion: The results from baseline (time zero) and after treatment (90-day) were analyzed by ANOVA followed by the Duncan test. $P$ values $<0.05$ were considered statistically significant. Group 1 presented the best clinical results and the best reduction in the microbiological index, with significance level $(\mathrm{p}<0.05)$. Group 2 had a significant improvement in clinical evaluation $(p<0.05)$, however, without significance in the microbiological index, when compared to Group 1. 
Placebo treatment (Group 3) was able to induce little improvement in the clinical parameters, but no significance in the microbiological index ( $p>0.05)$.

Conclusion: Biotherapy use (BP and auto-isotherapy) presented promising therapeutic potential in chronic periodontal diseases. In order to increase the confiability of these results, this protocol will repeat with a greater number of individuals.

Keywords: biotherapy; periodontitis; pilot study.

\section{References}

1. Siqueira, CM; Homsani, F; Veiga, VF; Lyrio, C. Matos, HJ; Passos, SRL; Couceiro, JN; Holandino, C. Homeopathic medicines for prevention of influenza and acute respiratory tract infections in children: blind, randomized, placebo-controlled clinical trial. Homeopathy, 105:71-77, 2016.

2. ANVISA. Agencia Nacional de Vigilancia Sanitária. Farmacopeia Homeopática Brasileira, 3a ed., Ed. ANVISA, Brasilia; 2011; pages 3133.

Received: March 1, 2108. Accepted: April 26, 2018.

(C) International Journal of High Dilution Research. Not for commercial purposes. 